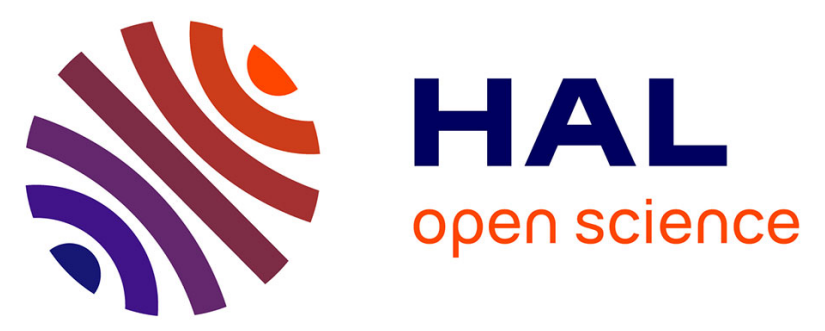

\title{
Delamination of multilayer graphene stacks from its substrate through wrinkle formation under high pressures
}

I.R. R Amaral, A. Forestier, A. Piednoir, R. Galafassi, Colin Bousige, Denis Machon, Olivier Pierre-Louis, R.S. S Alencar, A.G. G Souza Filho, Alfonso San-Miguel

\section{To cite this version:}

I.R. R Amaral, A. Forestier, A. Piednoir, R. Galafassi, Colin Bousige, et al.. Delamination of multilayer graphene stacks from its substrate through wrinkle formation under high pressures. Carbon, 2021, 185, pp.242-251. 10.1016/j.carbon.2021.09.029 . hal-03355812

\section{HAL Id: hal-03355812 \\ https://hal.science/hal-03355812}

Submitted on 27 Sep 2021

HAL is a multi-disciplinary open access archive for the deposit and dissemination of scientific research documents, whether they are published or not. The documents may come from teaching and research institutions in France or abroad, or from public or private research centers.
L'archive ouverte pluridisciplinaire HAL, est destinée au dépôt et à la diffusion de documents scientifiques de niveau recherche, publiés ou non, émanant des établissements d'enseignement et de recherche français ou étrangers, des laboratoires publics ou privés. 


\title{
Delamination of multilayer graphene stacks from its substrate through wrinkle formation under high pressures
}

\author{
I. R. Amaral ${ }^{a, b}$, A. Forestier ${ }^{\mathrm{a}}$, A. Piednoir ${ }^{\mathrm{a}}$, R. Galafassi ${ }^{\mathrm{a}}$, C. Bousige ${ }^{\mathrm{c}}$, D. Machon ${ }^{\mathrm{a}}$, O. Pierre-Louis ${ }^{\mathrm{a}}$, \\ R. S. Alencar ${ }^{\mathrm{d}}$, A. G. Souza Filho ${ }^{\mathrm{b}}$, A. San-Miguel ${ }^{\mathrm{a}, *}$ \\ ${ }^{a}$ Institut Lumière Matière, UMR5306 Université Lyon 1-CNRS, Université de Lyon F-69622 Villeurbanne cedex, France \\ ${ }^{b}$ Departamento de Física, Centro de Ciências, Universidade Federal do Ceará, CEP 60.455-970, Fortaleza, CE, Brazil \\ ${ }^{c}$ Laboratoire des Multimatériaux et Interfaces, UMR CNRS 5615, Univ. Lyon, Université Claude Bernard Lyon 1, F-69622 \\ Villeurbanne, France \\ ${ }^{d}$ Faculdade de Física, Universidade Federal do Pará, CEP 66.075-110, Belém, PA, Brazil
}

\begin{abstract}
There is a need to find new paths for van der Waals 2D-systems detachment and transfer or to control their adhesion state in different environments. We have observed that supported multilayer graphene immersed in a fluid can be detached from a substrate through pressure application. The process is based on the development of wrinkles originated by the difference of in-plane-compressibility between the graphene stacks and the substrate. Graphene stacks comprised between 9 and 110 layers and immersed in various fluids allowed to investigate the growth and evolution of wrinkles with increasing pressure. The detachment from the substrate stops at the pressure-induced fluid solidification. Methanol, ethanol or their mixtures favor the pressure-induced wrinkle formation in $\mathrm{SiO}_{2} / \mathrm{Si}$ substrates. In these cases, the pressure evolution of the delamination process follows a universal behavior independently of the number of graphene layers with a complete delamination at / sim4GPa. The quantitative analysis of the wrinkle geometry evolution can be consistently interpreted as due to a pressure-induced increase of the bending stiffness of the graphene stacks, or a reduction of the adhesion forces between the sample and the substrate, or both. These results should also be of practical use in high-pressure experiments of van der Waals systems.
\end{abstract}

Keywords: graphene, multilayer, high pressure, wrinkles, adhesion, mechanics

\footnotetext{
${ }^{*}$ Corresponding authors

Email address: alfonso.san-miguel@univ-lyon1.fr
}

(A. San-Miguel) 


\section{Introduction}

Two dimensional (2D) van der Waals (vdW) systems are frequently supported on a substrate or sandwiched in hererostructures which may determine their exact geometry and even impose electronic or chemical exchanges [1-3]. Different phenomena such as superconductivity, valleytronics, topological insulation or passivization, can benefit or be affected from the interactions between the $2 \mathrm{D}$-system with its substrate. As a consequence, methods to transfer 2D-systems onto different targeted substrates [4] or to produce stacks of van der Waals heterostructures [5] are the subject of intense investigation. In this context, finding new methods to control the interaction between a vdW system and its substrate - eventually allowing its transfer - could have a significant impact in many different fields.

Pressure application on such supported systems appears as an interesting way to continuously tune these interactions and better understand them $[6$, 7]. Graphene or graphene-based systems are particularly interesting in this regard due to their simple monoatomic nature and their relative ease of manipulation. In fact, pressure application has been shown to lead in graphene stacks to a combination of mechanical and chemical, e.g. doping, effects $[8,9]$, electronic structure evolution [10] or to structural transformations $[11,12]$.

The ability to induce changes with pressure in the mechanics or chemistry of these systems is expected to bring interesting applications in various fields such as tribology, composite materials or even straintronics - i.e. the tuning of the elec- tronic properties of a sample through mechanical strain. However, a better understanding of the mechanisms at play between graphene and its highpressure environment - its substrate and the pressure transmitting medium (PTM) - is highly desirable to reach the development of technological applications. From an experimental point of view, advanced techniques have been developed for studying graphene interaction with its environment at high pressure, especially using in situ Raman spectroscopy. Indeed, the Raman response of graphene is not only a very rich fingerprint of graphene crystallinity or stacking order determination, but it also provides information on mechanical deformations, adhesion on substrate and doping effects during the compression $[6,8,13,14]$. In particular, from the observation of the pressure evolution of Raman signatures, it has been proposed a possible detachment of graphene or bilayer graphene from its substrate at high pressure $[8,15]$. Nevertheless, no direct proof of such pressure-induced detachment has been provided up to now. While these questions have been raised for graphene or stacks of few layers [8], there is a lack of knowledge on the response of graphene thicker stacks.

$\mathrm{SiO}_{2} / \mathrm{Si}$ substrates have a particular place in the study of graphene and graphene based systems. First of all, they provide an interferencial-based contrast allowing for direct optical observation of graphene. Furthermore, the Raman signal can be enhanced through constructive interference $[16,17]$ in addition to Raman resonances due to the peculiar linear band structure of graphene near the K-point of the Brillouin zone $[18,19]$.

When submitted to high pressures, recent studies 
have shown that single and bilayer graphene undergo a biaxial compression transmitted from the substrate by interfacial adhesion $[8,13-15,20]$. In these studies, it was observed that only a fraction of the substrate's pressure-induced strain is transmitted to graphene, such as $\varepsilon_{G}(P)=\alpha \varepsilon_{S}(P)$ where $\varepsilon_{G}(P)$ and $\varepsilon_{S}(P)$ are the graphene in-plane biaxial strain of graphene and the substrate, respectively, and $\alpha$ is a phenomenological parameter that accounts for the incomplete strain transfer [13]. Similar observations were made in experiments on supported $\mathrm{MoS}_{2}$ at high pressure, where a bimodal adhesion state was probed [21]. The phenomenological parameter $\alpha$ summarizes the graphene-substrate adhesion, friction, graphene bending and ripple formation, the difference in bulk moduli between graphene and its substrate, substrate's roughness and commensurability.

The development of an elaborated, inhomogeneous strain field at the nanoscale due to rippling and/or sliding of graphene over the substrate has been proposed, providing in-plane strain dissipation during the compression mechanism [13]. Graphene buckling and crumpling under compression have indeed been reported in various experimental works on graphene mechanics [22-26]. In ref. 25, the buckling threshold identified corresponds to a compressive strain of $0.21 \%$ in the graphene sheet when graphene is on a $\mathrm{SiO}_{2} / \mathrm{Si}$ substrate. In the case of high pressure experiments, a value of $\varepsilon_{S}(P)=$ $0.21 \%$ at the $\mathrm{SiO}_{2} / \mathrm{Si}$ substrate surface is achieved at pressure as low as $\sim 0.7 \mathrm{GPa}$. Pressure induced graphene buckling, leads then to the local production of curved graphene and may be seen as the reciprocal effect of pressure induced carbon nan- otube radial collapse which leads to a local loss of curvature [27].

In this study, we assess such buckling, wrinkling, and out-of-plane effects in supported graphene samples loaded in a diamond anvil cell (DAC) pressure apparatus (Fig. 1(a-b)). In such experimental conditions, the sample surface imaging can be obtained using optical in situ probes which are intrinsically limited by the diffraction limit. Thus, only micrometer-scale and larger features can be observed by optical inspection and Raman spectroscopy, while wrinkling and rippling in monolayer graphene are expected to be considerably smaller, i.e. at nanometer scale [14]. This is the main reason why such out-of-plane effects have never been observed in high-pressure experiments on graphene, and were only suggested as the mechanism of partial strain transfer from the substrate. Here, we have chosen to study samples of nanometer thickness (few tens of layers) so that these out-of-plane deformations, wrinkles and buckling effects are likely to occur at a larger scale, mostly due to their larger bending stiffness. Our approach is to address these multilayer graphene samples on $\mathrm{SiO}_{2} / \mathrm{Si}$ substrate at high pressure, bringing easier visualization of the out-of-plane displacements by optical inspection thanks to the contrast enhancement effect [28] (Fig. 1(c)). It is important to note that the interaction between $n$-layered graphene samples and the $\mathrm{SiO}_{2} / \mathrm{Si}$ substrate strongly depends on the number of layers, as mentioned. A transition takes place for $n>2$, since the bending modulus of thicker systems is large enough to avoid its conformation on the $\mathrm{Si} / \mathrm{SiO}_{2}$ substrate [14]. All the studied samples in this work correspond to the $n>2$ case. In such 
(a)

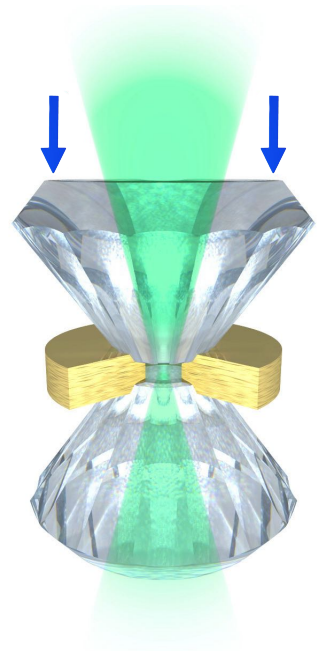

(b)

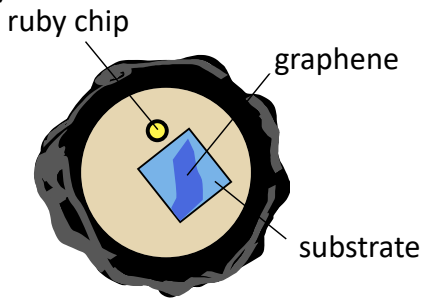

(c)

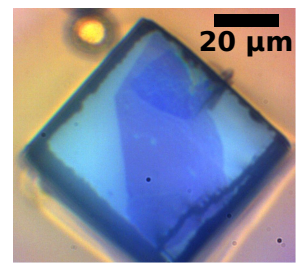

(d) $\mathbf{P}=\mathbf{0}$
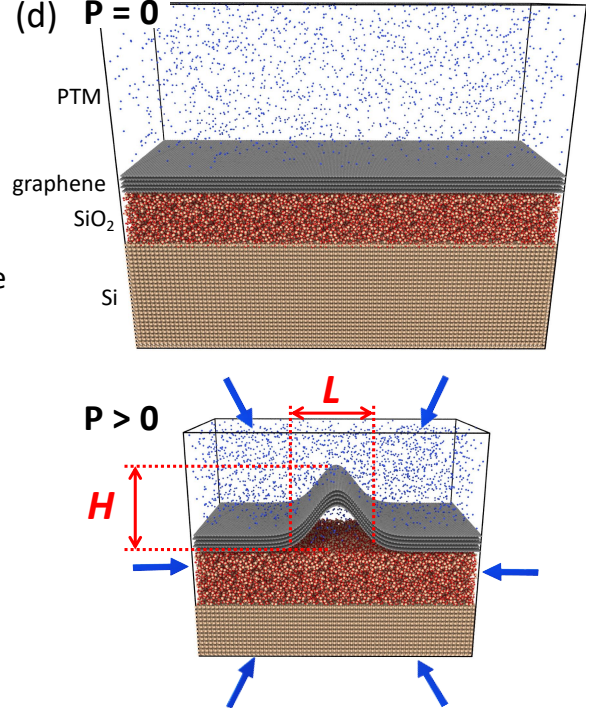

Figure 1: Schematics of the experiment. - (a) Sketch of the diamond anvil cell (DAC) pressure apparatus used to compress the sample. The two blue arrows represent the applied force to the diamond anvils which reduce the volume delimited by the hole at the center of the metallic gasket placed between the diamonds. The gasket is shown opened to illustrate the sample chamber. Optical access through the diamond anvils allow one to image and perform Raman spectroscopy or luminescence measurements. - (b) Drawing of the sample chamber in which the substrate supporting the multilayered graphene sample is immersed in a PTM fluid. A ruby chip is placed beside the substrate for the in situ measurement of the pressure through its luminescence. - (c) Optical image of a real sample used in experiments in which the substrate and a multilayered graphene sample are visible. A ruby chip sphere can be seen at the top. - (d) The sample consisting on $n$-layers of graphene lies on a substrate (upper image) made of silicon and a silicon oxide layer. Upon pressure application (lower image), the substrate's volume decreases, thus leading to the creation of wrinkles in the graphene layers. While liquid, the PTM is able to flow under these wrinkles, which are characterized by their height $H$ and width $L(\mathrm{~d})$, as discussed in the modeling section.

conditions, the PTM is susceptible to infiltration between the sample and the substrate surface eventually stabilizing wrinkles as schematically shown in Fig. 1(d). Therefore, both interactions with the substrate and with the PTM are expected to play an important role and will be deeply analyzed in our work. For this, we have performed multiple high-pressure experiments with different PTM commonly used in high pressure studies, such as nitrogen [9, 14], water [11], paraffin oil [8, 29], methanol, ethanol and their 4:1 mixture.

\section{Experimental}

Thin graphite samples were obtained by mechanical exfoliation from natural graphite (Naturgraphit $\mathrm{GmbH}$ ) using PDMS thin films [30]. After a few micro-cleavages, the resulting flakes are deposited on a PDMS stamp for later inspection and transfer. Optical contrast inspection allowed to locate flakes with appropriate thickness for our study (9 to 110 layers of graphene). Sample's thicknesses were then determined with $\sim 3 \%$ accuracy, using an atomic force microscope (AFM) MFP-3D (Asylum Research, Oxford Instruments) in tapping mode di- 
rectly on the flakes lying on PDMS.

The substrates used for high pressure experiments were obtained from a $50 \mu \mathrm{m}$ thick $\mathrm{Si} / \mathrm{SiO}_{2}$ wafer (raw silicon covered by $300 \mathrm{~nm}$ of thermal oxide). It was cut into small square pieces of $\sim 50$ $\mu \mathrm{m}$ side using a diamond saw to obtain sufficiently small pieces to be loaded.

The diamond anvil cells used in this work are membrane driven DAC [31] with $600 \mu \mathrm{m}$ anvil culet diameter (a DAC with $400 \mu \mathrm{m}$ anvil culet diameter was also used). Stainless steel gaskets with $\sim 200 \mu \mathrm{m}$ hole diameter and appropriate indentation thickness $(>50 \mu \mathrm{m}$, the thickness of the substrates) were used to properly confine the sample in the DAC (Fig. 1(a-c)).

The loading is performed in two steps: (i) a small square piece of $\mathrm{SiO}_{2} / \mathrm{Si}$ substrate is deposited and glued on the bottom diamond anvil, and (ii) the chosen multilayer graphene flake is transferred on its surface by deterministic dry transfer using a similar set-up to the one described in ref. 32. One or a few small ruby chips are added along with the sample for pressure calibration through its luminescence lines shift with pressure [33]. An image of the sample environment is shown in Fig. 1(c).

Various PTMs were used: pure methanol and ethanol, their 4:1 mixture, paraffin oil, distilled water, and nitrogen. In the latter case, the PTM loading in the DAC was performed by immersion of the cell in liquid $\mathrm{N}_{2}$ at $77 \mathrm{~K}$. All the other PTM used are liquid at ambient temperature and were loaded by drop deposition in the pressure chamber.

In situ Raman measurements were performed by using a homemade back-scattering setup based on a Princeton Instruments ACTON 2500i spectrom- eter. The excitation wavelength was $532 \mathrm{~nm}$. A 1800 grooves/mm diffraction grating with $500 \mathrm{~nm}$ blaze wavelength was used to disperse the scattered light. The microscope objective used for both Raman measurements and optical inspection is a long working distance objective (Mitutoyo Japan) with $50 \times$ magnification and 0.42 numerical aperture (in some experiments, a $100 \times$ objective with $\mathrm{NA}=0.7$ was also used). All pictures and videos were recorded using a Thorlabs DCC1645C CMOS camera installed on the Raman setup.

\section{Results}

Optical micrographs of a number of significant high pressure experiments on the multilayer graphene systems are shown in Fig. 2. The first seven columns (a to $\mathrm{f}$ ) of the figure correspond to experiments using $\mathrm{SiO}_{2} / \mathrm{Si}$ substrates and the last column (g) to an experiment using diamond as substrate. The number of graphene layers in each experiment was determined prior to the high pressure experiment using AFM.

Fig. 2(a) corresponds to optical micrographs obtained for different pressures in a multilayer graphene sample having a measured thickness of $\sim 17 \mathrm{~nm}$, corresponding to approximately 51 graphene layers [34]. Fig. S1(a) of supporting information (SI) shows the AFM scan for this precise sample as well as the height profile across its edge. The 4:1 methanol:ethanol mixture was used as PTM for reference, since it has the remarkable property to remain liquid up to $\sim 10 \mathrm{GPa}[35-37]-$ thus ensuring pure hydrostatic compression inside the DAC up to this pressure. 


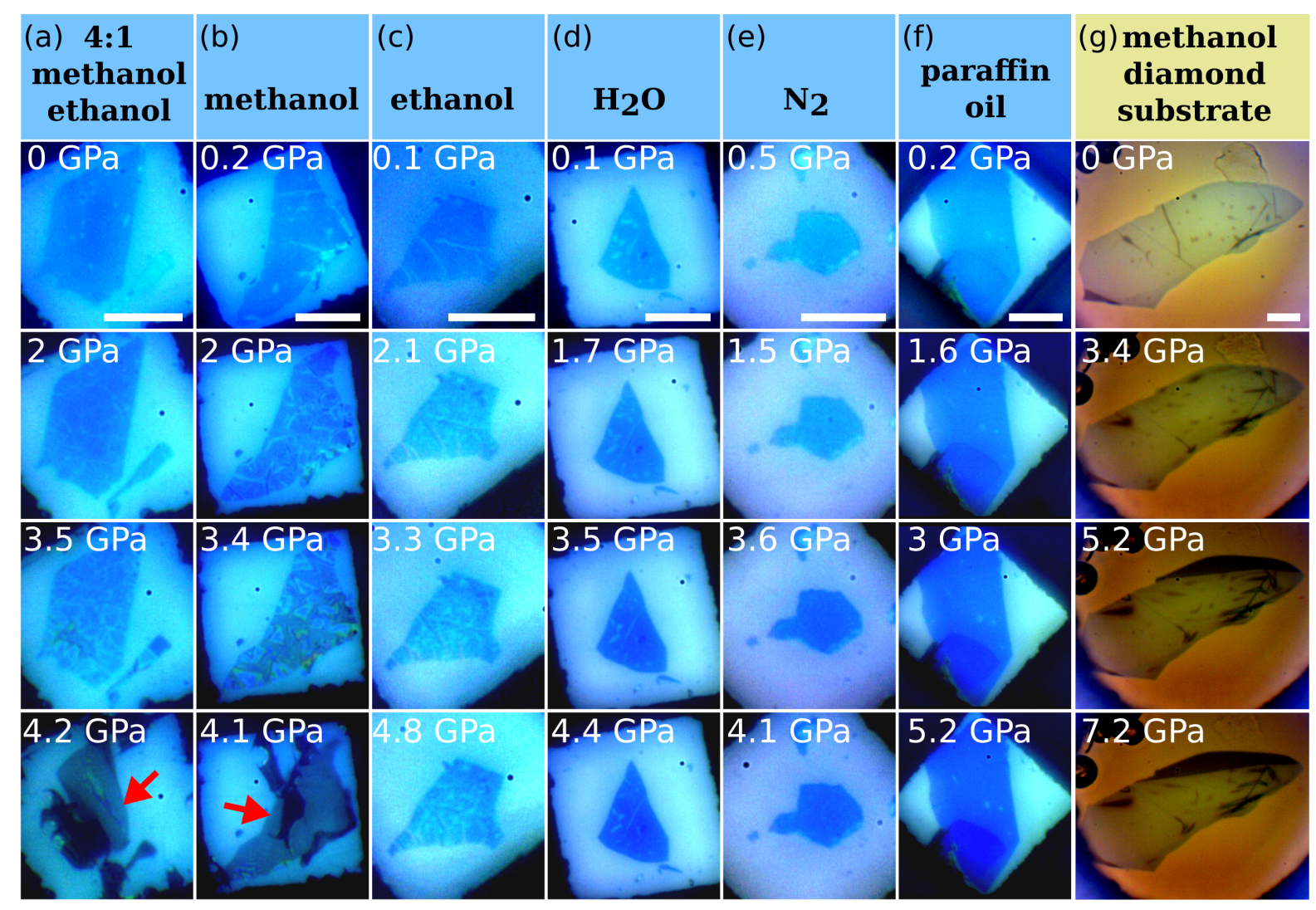

Figure 2: Optical micrograph of supported multilayer graphene samples under high pressure. Each column shows images for an experiment done with different PTMs indicated at the top of the column. All experiments were done on $\mathrm{SiO}_{2} / \mathrm{Si}_{\text {substrates }}$ (light blue square) except in the last column corresponding to a diamond substrate. The measured number of layers of the multilayer graphene samples are from left to right: $51 \pm 4,30,30,30,24,21,48 \pm 9$. The development of wrinkles with applied pressure is clearly seen in the first 3 columns. Red arrows point to the totally detached multilayer graphene observed in two experiments. White scale bars correspond to $20 \mu \mathrm{m}$ on every micrograph.

The sample is clearly identifiable on the $\mathrm{SiO}_{2} / \mathrm{Si}$ substrate surface by optical contrast - it corresponds to the darker blue color. When the sample is observed by in situ optical microscopy in the DAC, the contrast is lower than for ambient conditions, mainly due to the presence of the alcohol PTM and the diamond anvil introducing optical aberrations. Thus, in order to help identify optical effects on the surface of the sample with pressure increase, the optical contrast was numerically in- creased in all the obtained images. Looking closely at Fig. 2(a), light blue lines on the multilayer sample surface are observed and develop with pressure increase. This effect is particularly strong at higher pressures ( $3.5 \mathrm{GPa}$, Fig. 2(a)), where optical interferences are also observed in the lower region of the sample.

A complete detachment of the sample from the substrate is finally observed in the last micrograph in Fig. 2(a) (corresponding to $P=4.2 \mathrm{GPa}$ ). In- 
deed, in this last picture, no blue optical contrast enhancement from the $\mathrm{SiO}_{2} / \mathrm{Si}$ substrate is observed anymore, and the flake appears dark, thus indicating its complete unbinding from the substrate. This detachment effect is not reversible with pressure decrease, and we often have observed the flake floating above the substrate while applying pressure in the few GPa range (see video V1 in SI). Most of the time, the flake is lost when the DAC is opened at the end of the pressure cycle, or it ends up lying on the diamond anvil. This floating is an ideal situation when running hydrostatic pressure experiments with the fluid completely surrounding the $2 \mathrm{D}$ system.

Logically, the progressive observation of lighter blue lines on the sample surface at lower pressures corresponds to regions where the multilayer flake detaches from the substrate. It is thus reasonable to suggest that it corresponds to the formation of wrinkles in the multilayer flake. At these wrinkle positions over the $\mathrm{SiO}_{2} / \mathrm{Si}$, different optical interference conditions are matched, which leads to the lighter blue color observed. In brief, pressure application on the supported multilayer graphene sample using 4:1 methanol:ethanol as PTM leads to (i) its progressive detachment from the substrate through the formation of wrinkles, and ultimately (ii) to its complete detachment from the underlying substrate, near $\sim 4 \mathrm{GPa}$.

We may note that in the last picture in Fig. 2(a), black regions can be seen on the $\mathrm{SiO}_{2} / \mathrm{Si}$ substrate surface. This effect was also observed by compression of the substrate alone, without any graphene sample. This corresponds to the fracture of the $\mathrm{SiO}_{2}$ oxide layer from the underlying $\mathrm{Si}$ wafer due to the different strain rates of these two materials with pressure, as already observed in previous work [9]. Indeed, silicon's bulk modulus is three times larger than the one of the oxide layer, leading to the breakdown observed due to interfacial stress $[38,39]$.

To determine whether similar wrinkling and delamination of the multilayer graphene samples are observed at high pressure using other PTMs, experiments using (i) distilled water, (ii) nitrogen and (iii) paraffin oil as PTMs were performed. Micrographs obtained at high pressure are shown in Fig. 2(d), (e), and (f), respectively. The thicknesses of these investigated are 8,10 and $7 \mathrm{~nm}$, respectively.

For these three PTM studied, no comparable wrinkle formation nor complete detachment of the flakes from the substrate were observed, despite reaching pressures similar to the first experiment with alcohol (>4 GPa). In the case of water PTM, the detachment regions are visually faint but still present in the lower pressure domain. However, its extent is nothing similar to the alcohol PTM case in which a complete detachment occurs.

It is important to consider the different properties of the PTMs, particularly their solidification pressure at ambient temperature. Water as well as nitrogen crystallize at relatively low pressures, 1 and 2.4 GPa, respectively [40, 41]. Paraffin oil, on the contrary, is reported to undergo complete solidification at pressure as high as $7 \mathrm{GPa}$ [42], with a significant increase in viscosity at ambient temperature in this pressure range [43]. During our experiments using paraffin oil as PTM, we did not observe any sign of non-hydrostatic conditions in the 
pressure evolution of the ruby luminescence lines shifts and widths up to $5.2 \mathrm{GPa}$, the higher pressure reached. Even if the state (liquid or solid) of the PTM plays an important role as we will discuss later, it is clearly not enough to explain our observations. Indeed, using nitrogen as PTM, no wrinkling or detachment are observed even in its liquid state regime (in the $0-2.4 \mathrm{GPa}$ range), while it is clearly observed within the same pressure range when the alcohol mixture PTM is used.

Since wrinkling and full delamination are observed solely in 4:1 methanol:ethanol, we performed a sequential comparative in situ Raman measurements of the Raman G-band from a supported multilayer graphene sample. We used nitrogen PTM in a first pressure cycle and then, in a second one on that same sample, it was used 4:1 methanol-ethanol PTM. The goal of these measurements was to determine whether the mechanical strain at the atomic bond scale could be different in these two cases, since, as already discussed, the optical contrast observations strongly differ. The G-band evolution as a function of pressure in both cases can be seen in the Fig. S1(b) in SI. Results for both PTMs are very close to the bulk graphite response. This means that there is no biaxial strain transfer at the atomic scale [13]. This is somewhat expected for stacks with more than two graphene layers [14], i.e., for all our samples. Consequently, in the present case, Raman spectroscopy is not a good probe to evidence an evolution in the macroscopic adhesion between the graphene stacks and the substrate. A slightly stronger shift for nitrogen PTM is observed in the higher pressure points, that may be due to a small strain transfer contribution arising from the solidified nitrogen [9].

To better investigate the favoured unbinding effect in the alcohol mixture PTM, we performed high pressure runs on two samples: one in pure methanol as PTM, and the other in pure ethanol. Optical pictures obtained at high pressure are shown in Fig. 2(b) and (c), respectively. In the case of pure ethanol PTM (Fig. 2(c)), the sample wrinkling effect is observed through the rising of light blue channels under the sample, but no complete detachment is found at higher pressures up to 4.8 GPa. Even more, one can see in the images that beyond 3.3 GPa, there is a gradual re-adhesion of the sample on the substrate, observed as the disappearance of some of the light blue wrinkles. When pure methanol was used as PTM (Fig. 2(b)), the effect was more pronounced with a total detachment of the flake near $4 \mathrm{GPa}$.

It is important to mention that ethanol undergoes a transition from its liquid phase to a solid monoclinic crystal structure near $\sim 1.9 \mathrm{GPa}[44]$. Pure methanol, on the other hand, is expected to undergo a gradual crystallization starting near $3.5 \mathrm{GPa}$, but is commonly found in a superpressed (metastable) liquid state in the 3.5-10.5 GPa range due to its low symmetry and the strained hydrogen bonds of its molecules [37, 44-48]. In fact, to experimentally achieve crystallization of methanol, a slow pressure cycling above the crystallization pressure is required [48]. In our experiments, we always achieved the superpressed liquid phase (see Fig. S2 in SI).

We have summarized our observations in Fig. 3(a) by representing the pressure evolution of the ratio between unbound area of the sample over 
(a)
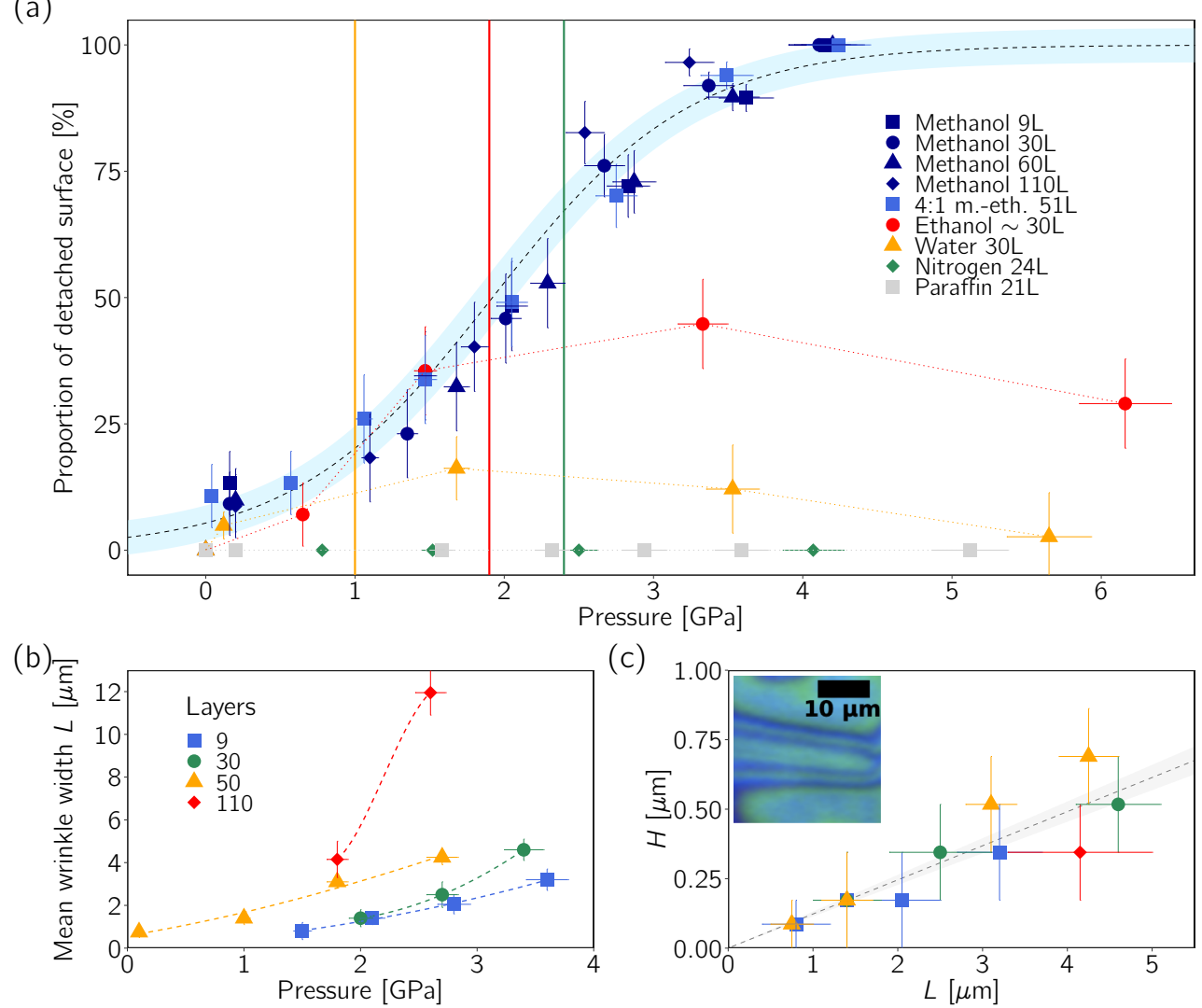

(c)

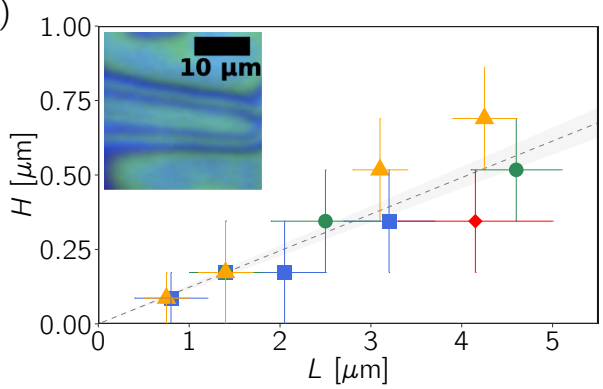

Figure 3: Wrinkles geometry evolution with pressure. (a) Pressure evolution of the proportion of the detached surface of the samples for all experiments performed (see legend). The light blue curve is a sigmoidal fit of the data obtained for pure methanol PTM, intended as a guide to the eye. Vertical yellow, red and green lines indicates the solidification pressures of water, ethanol and nitrogen, at respectively $1 \mathrm{GPa}, 1.9 \mathrm{GPa}$ and $2.4 \mathrm{GPa}$. Two extreme definition rules for the boundaries of the detached surface were used to define the error bars (see Fig. S3 in SI). (b) Average wrinkle width $L$ as a function of pressure for samples with various thicknesses in methanol PTM. Dotted lines are guides to the eye, and error bars correspond to the standard deviation of the measured wrinkle widths. (c) Measured wrinkle height $H$ as a function of pressure corresponding to the points in (b) except one of the points which was not possible to measure (same symbol code as in (b)). The $H$ values were evaluated from the interference fringes of visible light coming from the microscope (full details are found in SI). The inset shows the optical interference pattern of a wrinkle in a region of a 110L-graphene used to determine the height. The dotted line is a linear fit of the data.

its total area, i.e. the proportion of the peeled off carbon sample. This proportion was evaluated by image analysis, and two extreme definition rules for the boundaries of the detached surface were used to define the error bars in Fig. 3(a) - see Fig. S3 in SI for details. Note that Fig. 3(a) includes experi- ments on flakes of various thicknesses ranging from 9 to 110 layers using pure methanol PTM, for which the corresponding optical micrographs are given in the Fig. S4 in SI.

Looking at Fig. 3(a), an interesting trend emerges: when the PTM is either pure methanol or 
4:1 methanol:ethanol mixture, all data points follow the same increasing tendency with pressure, highlighted by a light blue band. This general trend shows a progressive unbinding process which can be well described by a logistic function independently of the sample thickness from 9 to 110 graphene layers. In Fig. 3(b) we show the measured average width, $L$, of the observed wrinkles as function of pressure for samples of different thickness in methanol. The thicker the sample, the larger the observed wrinkles. As can be observed in Fig. 3(c), also the wrinkle height, $H$, increases with pressure, but, with our precision, its evolution appears to be independent of the sample thickness. Combining the results of Fig. 3(a), (b) and (c) this leads to the conclusion that the surfacic density of wrinkles depends on the sample thickness, the wrinkles density being the largest for the thinner samples where the detachment is obtained through the formation of a field of thinner wrinkles.

On the other hand, Fig. 3(a) shows that for ethanol and water PTM, the data points start to follow this same trend up to the point of PTM solidification, above which no further evolution is observed within measurement errors. In the case of nitrogen and paraffin oil, no wrinkling or detachment is observed at all. These observations show that (i) the pressure evolution of the proportion of detached surface does not significantly depend on the flake's thickness, and that (ii) the observed detachment is not only governed by the mechanical response to pressure but also by a specific chemical interaction between the PTM molecules and the multilayer graphene sample.

When wrinkles appear under pressure and while the PTM is fluid, we expect the PTM to immediately adsorb along the newly created nanoporous channels. Much like what is observed at low temperature[49-52], high pressure brings the PTM molecules close together and favors the formation of long adsorbed zigzag chains under the graphene surface. Together with the effect of pressure, this chemical interaction results in wrinkle expansion, leading to the flake's ultimate detachment, as long as the PTM remains liquid.

\section{Discussion and modelling}

Our observations of pressure-induced wrinkling and detachment of the multilayer graphene sample in alcohol PTM may appear as counter intuitive. Indeed, the reduction of the graphene-substrate distance due to the perpendicular force applied to the system - leading to a stronger adhesion between them - is intuitively expected $[8,53]$, in opposition to the observed unbinding. In the following paragraphs, we will analyze the details of the wrinkle pattern formation both from our experimental data and through modelling in order to provide an explanation to this unexpected behavior. The substrate and the PTM physical properties will play a central role.

First of all, to understand the origin of wrinkle formation, we must consider the difference in compressibility between the nanometric thin graphite sample and the substrate, characterized by their respective bulk moduli. While the graphite inplane linear bulk modulus is very large, $-r \partial P / \partial r \sim$ $1250 \mathrm{GPa}$, the silicon substrate's linear bulk modulus is approximately four time smaller, i.e. 
$294 \mathrm{GPa}[39,54]$ ( $r$ denotes the inter-atomic distance). In other words, at a given pressure, the linear strain at the substrate surface is four time larger than the in-plane strain of bulk graphite.

Our in situ Raman measurements on the multilayer samples show a similar evolution to graphite, indicating that no additional strain is induced in the sample by adhesion on the substrate as it was observed for the thinnest samples of one or two layers in our previous works $[8,9,13,14]$. However, the multilayer sample is still lying on the substrate (at least initially) and is likely to be displaced by means of adhesion on the compressed substrate at some specific regions or points. These displacements are likely to be dissipated through out-ofplane displacements of the membrane, leading to the observed wrinkling. Similar wrinkling effects were observed in several previous works in which graphene was strained through thermal expansion mismatch with the substrate [24, 25], or through the use of a flexible polymer substrate or matrix $[23,26]$.

This first mechanical consideration can explain the formation of wrinkles, but does not explain why these are observed with pure methanol PTM (as well as with pure ethanol in the low pressure regime) but not with other PTMs, such as water, nitrogen or paraffin oil. An intuitive criterion to examine the formation of wrinkles is the physical state of the PTM used. Indeed, a solid PTM at the sample surface may hinder the formation of wrinkles, preventing the membrane from buckling in the perpendicular direction. A liquid PTM, however, allows for such buckling and may even be able to diffuse between the sample and the substrate, inside the channels created by the wrinkles.

This hypothesis explains the formation of wrinkles with water and pure ethanol PTM that stops at higher pressures (water crystallizes at $\sim 1 \mathrm{GPa}$ and ethanol at $1.9 \mathrm{GPa}$ ), and more prominently, the absence of final complete detachment (Fig. 3(a)). On the other hand, neither wrinkles nor detachment are observed in paraffin oil and nitrogen, although paraffin oil, despite its increasing viscosity, remains liquid over the whole studied pressure range, and nitrogen is liquid up to $2.4 \mathrm{GPa}$. Thus, the PTM solidification is not enough to explain all observations.

Our observations can thus be understood by considering the following process: (1) the in-plane bulk modulus mismatch between the multilayer graphene and its substrate leads to the buckling of the graphene membrane as pressure increases, and (2) for some liquid PTM, its molecules diffuse into the channels created by this buckling, enlarging them and making such wrinkles directly observable by optical inspection. (3) This process stops when the PTM solidifies or when the 2D flake is fully detached from its substrate. The fact that the PTM is liquid is however not sufficient to observe (2), as for liquid PTM like paraffin oil and nitrogen, the phenomenon was not observed. Therefore, the alcohol-graphene interaction at high pressure appears to show a singular behavior with respect to the other tested PTM. We however emphasize here that wrinkles do occur in each case, even in nitrogen and paraffin oil PTM. However, it is only due to the described insertion of PTM molecules under the graphene flake and the consecutive enlargement of the wrinkles (e.g. alcohol mediums), 
that we are able to see them through optical microscopy. Indeed, narrow initial wrinkles were visible on AFM topographic images of the flakes on PDMS, but were not resolved by optical microscopy after sample transferring onto $\mathrm{SiO}_{2} / \mathrm{Si}$ (see Fig. S5 in SI).

With this process, one can understand that the sample thickness plays a minor role in our observations: the interaction between the adsorbed PTM molecules inside the wrinkles channels (under the sample) is a surface effect, which is not significantly affected by the sample's thickness. Despite the increasing wrinkle width related to the increasing bending rigidity due to the number of layers [55] (Fig. 3(b)), a complete detachment is always visible at approximately $4 \mathrm{GPa}$ (see Fig. S4 in SI). Because the adsorption occurs on the outer external graphite layers, the number of layers would not significantly influence the adsorption energy on the surface layer, which is responsible for the sample delamination.

The chemical dependence of our observations can be explained by the fact that the methanolgraphene interaction is stronger than the methanolmethanol intermolecular interaction itself. This particularity leads to the preferential formation of a full adsorbed methanol layer before the stacking of supplemental adsorbed methanol layers on the graphite surface $[56,57]$. This strong interaction may explain why the infiltration of methanol inside wrinkles under the flake leads to the expansion of the channels and the flake's complete detachment, while it is not the case with liquid nitrogen and paraffin oil.

In order to test our hypothesis, we have per- formed a complementary high pressure experiment in which the multilayer flake was directly transferred onto the diamond anvil rather than on an intermediate substrate, using pure methanol as PTM. The sample thickness was $16 \pm 3 \mathrm{~nm}$ (48 \pm 9 layers). The linear bulk modulus of the diamond substrate, $\sim 1330 \mathrm{GPa}$, is very close to the in-plane bulk modulus of graphite [13], and therefore only a very weak wrinkling is expected. As one can see in Fig. 2(g), the detachment is observed mostly at the flake edges and at an even higher pressure (starting from 5.2 GPa). This observation is in clear agreement with our explanation of the wrinkling and detachment mechanism mediated by the insertion of the alcohol PTM beneath the flake.

To gain a deeper understanding on the physical processes involved in pressure-induced wrinkle formation and the observed unbinding, let us now consider a physical quantitative analysis. Wrinkles form to relieve stretching energy caused by the contraction of the substrate via a well-known scenario [59]. When stretching energy is relieved, the equilibrium shape of one wrinkle mainly results from a compromise between adhesion energy and bending energy. In addition, the network of wrinkles is connected and always reaches the edges of the flakes. Hence, we assume that the liquid PTM can freely flow below the graphene into the wrinkles, so that there is no volume constraint under them. Furthermore, since the observed wrinkle height is much smaller than their width, we use the small slope approximation. The wrinkle profile $h(x)$ as a function of the coordinate $x$ orthogonal to its direction is then obtained by a straightforward minimization of 
(a)

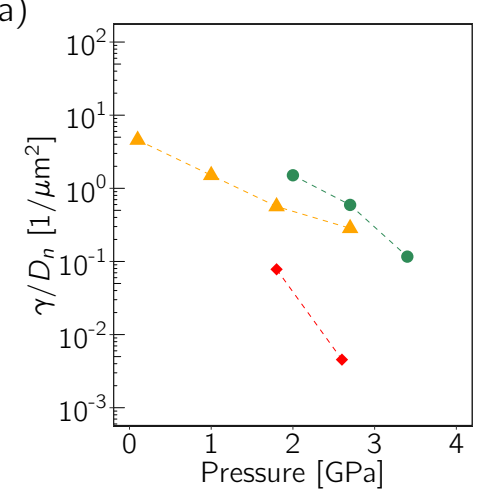

(b)

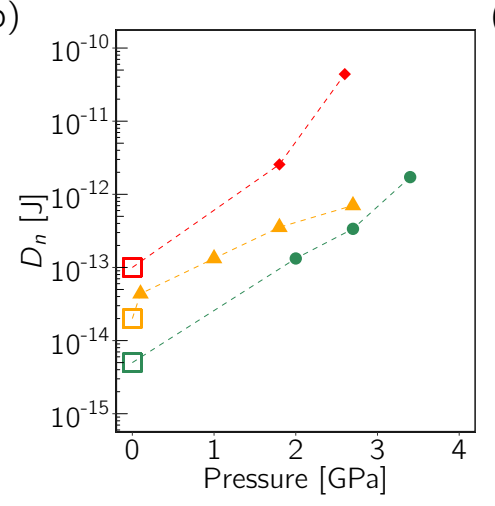

(c)

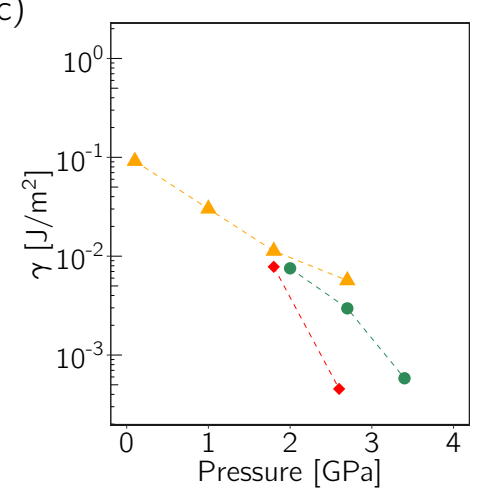

Number of layers $\bullet 30-50 \bullet 110$

Figure 4: Pressure evolution of the physical parameters involved in wrinkle formation for samples with a number of graphene layers, $n$, equal to 30 (green), 50 (orange) and 110 (red). (a) $\gamma / D_{n}$ ratio as a function of the pressure $P$ as obtained from eq. (2). - (b) Pressure evolution of the bending rigidity $D_{n}$ when assuming a constant adhesion energy $\gamma=0.2$ J.m ${ }^{-2}$. The empty symbols correspond to the expected zero-pressure $D_{n}^{0}$ values [58]. - (c) Pressure evolution of $\gamma$ assuming an additive layer-dependent $D_{n}^{0}[58]$. Dashed lines are guides to the eye. Error bars were omitted due to the logarithmic scale.

the adhesion and bending energies, such as $[60,61]$

$$
h(x)=\frac{H}{2}\left[1+\cos \left(2 \pi \frac{x}{L}\right)\right],
$$

where $L$ and $H$ are the width and height of the wrinkle, respectively (see Fig. 1(d)). Energy minimization also imposes a relation between $H$ and $L$ [61]:

$$
\frac{\gamma}{D_{n}}=2 \pi^{4} \frac{H^{2}}{L^{4}}
$$

where $\gamma$ is the adhesion energy per unit area and $D_{n}$ is the bending rigidity of $n$-layer graphene. We do not expect $\gamma$ to depend on the number of layers as it corresponds to the interaction of the layer immediately in contact with the substrate.

In Fig. 4(a), a plot of $\gamma / D_{n}$ as a function of pressure is obtained from Eq. (2) using our measurements of $L$ and $H$ on the same wrinkle (Fig. 3(bc)). Results are obtained for samples corresponding to 30,50 and 110 graphene layers. They show a striking decrease of the ratio $\gamma / D_{n}$ by a factor of more than 10 when the pressure is increased up to $\sim 3-4 \mathrm{GPa}$. This could originate from a decreasing $\gamma$, an increasing $D_{n}$ or a combination of both effects.

An increase of $D_{n}$ under pressure could be induced by a decrease of the sliding between the individual graphene sheets[58] within the multi-layer graphene. This scenario is supported by the observation of the stacking faults formation in multilayer graphene in strained nanocomposites [62], which could participate to the inter-monolayer sliding. Such movement could be decreased under pressure due to the work required to separate the graphene layers during sliding in the presence of an atomic corrugation between the sheets. Since the energy of stacking faults per unit area in Bernal stacking [62] $\left(\sim 0.2 \mathrm{meV} /\right.$ atom $\sim 10^{-1}{\mathrm{~J} . \mathrm{m}^{-2}}^{-2}$ is similar to the energy per area to separate two graphene sheets by $1 \AA$ under a pressure of $1 \mathrm{GPa}$, the formation of dislocations can be strongly influ- 
enced by the pressure.

The adhesion energy of monolayer graphene with a silicon oxide substrate in vacuum is about $[63] \gamma \simeq$ $0.45 \mathrm{~J}^{-2}{ }^{-2}$. For multi-layer graphene in vacuum,

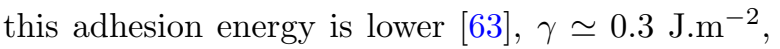
due to partial unbinding [14]. Immersed in a liquid media, the adhesion energy of multi-layer graphene on a silicon oxide substrate is expected to be even smaller by about $40 \%$ [64], and a reasonable value for ethanol medium at zero pressure is therefore $\gamma \simeq 0.2{\mathrm{~J} . \mathrm{m}^{-2}}^{2}$. Using Eq. (2), $D_{n}$ is plotted in Fig. 4(b) by assuming this latter value for $\gamma$, and by considering it to be independent of pressure and the number of graphene layers. This figure reports the resulting increase of the bending rigidity $D_{n}$ with pressure and with the number of layers. The plotted values are to be compared with the expected values of the bending rigidity at zero pressure, $D_{n}^{0}$, which were extracted from AFM measurements on graphene bubbles of varying thickness $\operatorname{as}[58] D_{30}^{0} \simeq 0.5 \cdot 10^{-14} \mathrm{~J}, D_{50}^{0} \simeq 2 \cdot 10^{-14} \mathrm{~J}$, and $D_{110}^{0} \simeq 10 \cdot 10^{-14} \mathrm{~J}\left(\right.$ with $\left.D_{1}^{0} \simeq 2.7 \cdot 10^{-19} \mathrm{~J}[65,66]\right)$. It is important to underline that there is nevertheless an important spread in propositions for $D_{1}^{0}$ values $[67,68]$. The evolution of $D_{n}(P)$ shown in Fig. 4(b) is in agreement with these extrapolated values (empty symbols). In summary, this first scenario assumes an increase of the bending modulus due to a decreased amount of interlayer sliding (i.e. increased interlayer friction) when the pressure is increased.

The other scenario is that of a decrease of the adhesion energy $\gamma$. This second scenario is strongly supported by the fact that the increase of the wrinkle width finally leads to complete unbinding of graphene with no subsequent reattachment of the multi-layer graphene to the substrate. We may here consider in that case that the evolution of $\gamma$ with pressure represents the effective macroscopic adhesion energy, which may integrate the formation under pressure of non-visible wrinkles at the submicrometric scale.

In this second scenario, we may assume that the bending rigidity $D_{n}$ is constant over the pressure range for a given sample thickness. Using the layerdependent zero-pressure values $D_{30}^{0}, D_{50}^{0}$ and $D_{110}^{0}$ defined before in Eq. (2), we plot in Fig. 4(c) the pressure evolution of the adhesion energy $\gamma(P)$, for the three measured sample thicknesses. This figure shows that the $\gamma(P)$ evolution trend is independent of the sample thickness, and the extrapolated value at vanishing pressures is consistent with the

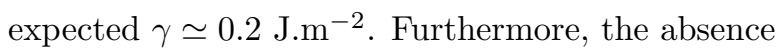
of re-attachment suggests that the effective adhesion energy has vanished or has become very small. However, even without a decrease of the adhesion energy, the absence of re-adhesion could be interpreted as due to the presence of the liquid medium PTM between the substrate and the graphene interface making the re-adhesion difficult. Indeed, the adhesion forces have to overcome the lubrication forces due to the evacuation of the viscous liquid PTM between the flake and the substrate to re-adhere. We may note here that a scenario corresponding to a combined evolution of $D_{n}$ and $\gamma$ can be equally advanced.

Our observations may then be extended to more compressible substrates, as polymers which are frequently used in graphene studies. Combination of mechanical and wetting methods could then ap- 
pear as possible new paths for van der Waals 2D systems detachment and transfer to other substrates [69]. Our results may also contribute to better understand the response of graphene based coatings in different environments submitted to mechanical constraints as in their use in tribological contacts $[70-72]$.

\section{Conclusions}

We have experimentally shown that thin graphite stacks with thickness varying between 9 and 110 layers can be detached from a silicon oxide substrate through pressure application. The used PTM plays an important role and, in particular, full delamination was only reached if the PTM could be maintained in its liquid state up to $\sim 4 \mathrm{GPa}$. Under these conditions, the thin graphite layer detaches progressively through the formation of a field of wrinkles whose total surface area progresses with pressure according to a universal sigmoidal curve, independently of the thickness of the sample. In the case of PTM pressure-induced solidification, the detachment from the substrate is stopped. We also found that methanol, ethanol or their mixtures favor the pressure-induced wrinkle formation in $\mathrm{SiO}_{2} / \mathrm{Si}$ substrates. The height of the wrinkles increases linearly with pressure and, within experimental precision, its evolution is also independent from sample thickness. The width of the wrinkle was also observed to increase with pressure, exhibiting higher values for thicker samples. Consequently, the surface density of the measured wrinkles at a given pressure is bigger for the thinner samples. Our observations lead to a significant pressure de- crease of the ratio between the effective adhesion energy and the sample bending modulus, $\gamma / D_{n}$ by a factor of $\sim 5-20$ per GPa. Both scenarios of an increase of $D_{n}$ and of the decrease of $\gamma$ appear to be quantitatively consistent. Finally, we have shown that the wrinkle area is a function of pressure, and that the wrinkle geometry at that pressure depends on the number of graphene layers. This offers opportunities to control the wrinkle average radius of curvature and its extension, which may impact optical [73] or chemical properties [74] to a great extent.

\section{Aknowledgements}

The authors thank thank Jérôme Degouttes (CNRS, INL Lyon) for technical support in silicon wafer cutting. This study was financed in part by the Coordenação de Aperfeiçoamento de Pessoal de Nível Superior - Brasil (CAPES) - Finance Code 001. The authors acknowledge the support from French-Brazil cooperation program CAPESCOFECUB project Ph938/19, 88881.192341/201801 and 88887.423558/2019-00. We acknowledge the support from CNRS projet "Défi-Biomimétisme" 2019 through project GPEG and for the ANR AAPG2019 support through project 2D-PRESTO. We acknowledge support from the iMUST LABEX program MUSCAT-2D. This work has been done thanks to the support of the high-pressure PLECE platform of the University of Lyon. R.S.A. also acknowledges funding from $\mathrm{CNPq}$ (Grant No. 311616/2020-8). A.G.S.F acknowledges funding from CNPQ (Grant No. 438144/2018-0, 309309/2017-4 and 442577/2019-2) and FUNCAP. 


\section{References}

[1] B. Li, J. Yin, X. Liu, H. Wu, J. Li, X. Li, W. Guo, Probing van der waals interactions at two-dimensional heterointerfaces, Nature Nanotechnology 14 (6) (2019) 567-572. doi:10.1038/s41565-019-0405-2.

[2] P. Ares, Y. B. Wang, C. R. Woods, J. Dougherty, L. Fumagalli, F. e. a. Guinea, Van der waals interaction affects wrinkle formation in two-dimensional materials, Proceedings of the National Academy of Sciences 118 (14) (2021) 2025870118. doi:10.1073/pnas . 2025870118.

[3] A. W. Huran, H.-C. Wang, A. San-Miguel, M. A. L. Marques, Atomically thin pythagorean tilings in two dimensions, The Journal of Physical Chemistry Letters (2021) 4972-4979doi:10.1021/acs.jpclett.1c00903.

[4] KimChan, YoonMin-Ah, JangBongkyun, KimJaeHyun, KimKwang-Seop, A review on transfer process of two-dimensional materials, Tribology and Lubricants 36 (1) (2020) 1-10.

[5] S. Fan, Q. A. Vu, M. D. Tran, S. Adhikari, Y. H. Lee, Transfer assembly for two-dimensional van der waals heterostructures, 2D Materials 7 (2) (2020) 022005 doi:10.1088/2053-1583/ab7629.

[6] Y. W. Sun, D. G. Papageorgiou, C. J. Humphreys, D. J. Dunstan, P. Puech, J. E. e. a. Proctor, Mechanical properties of graphene, Applied Physics Reviews 8 (2) (2021) 021310. doi:10.1063/5.0040578

[7] B. Sundqvist, Carbon under pressure, Physics Reports 909 (2021) 1-73. doi:https://doi.org/10.1016/j. physrep.2020.12.007

[8] D. Machon, C. Bousige, R. Alencar, A. Torres-Dias, F. Balima, J. e. a. Nicolle, Raman scattering studies of graphene under high pressure, Journal of Raman Spectroscopy 49 (1) (2018) 121-129. doi : 10.1002/jrs.5284.

[9] A. Forestier, F. Balima, C. Bousige, G. D. S. Pinheiro, R. Fulcrand, M. e. a. Kalbáč, Strain and PiezoDoping Mismatch between Graphene Layers, Journal of Physical Chemistry C 124 (20) (2020) 11193-11199. doi:10.1021/acs.jpcc.0c01898.

[10] F. Ke, Y. Chen, K. Yin, J. Yan, H. Zhang, Z. e. a. Liu, Large bandgap of pressurized trilayer graphene, Proceedings of the National Academy of Sciences 116 (19) (2019) 9186-9190. doi:10.1073/pnas.1820890116.
[11] L. G. P. Martins, M. J. S. Matos, A. R. Paschoal, P. T. C. Freire, N. F. Andrade, A. L. e. a. Aguiar, Raman evidence for pressure-induced formation of diamondene, Nature Communications 8 (1) (2017) 96. doi:10.1038/s41467-017-00149-8.

[12] L. G. Pimenta Martins, D. L. Silva, J. S. Smith, A.Y. Lu, C. Su, M. H. et al, Hard, transparent, sp3containing $2 \mathrm{~d}$ phase formed from few-layer graphene under compression, Carbon 173 (2021) 744-757. doi: 10.1016/j. carbon. 2020.11.038.

[13] C. Bousige, F. Balima, D. Machon, G. S. Pinheiro, A. Torres-Dias, J. Nicolle, D. e. a. Kalita, Biaxial strain transfer in supported graphene, Nano Letters 17 (1) (2017) 21-27. doi:10.1021/acs.nanolett.6b02981.

[14] J. Nicolle, D. Machon, P. Poncharal, O. Pierre-Louis, A. San-Miguel, Pressure-mediated doping in graphene, Nano Letters 11 (9) (2011) 3564-3568. doi:10.1021/ n1201243c.

[15] K. Filintoglou, N. Papadopoulos, J. Arvanitidis, D. Christofilos, O. Frank, M. e. a. Kalbac, Raman spectroscopy of graphene at high pressure: Effects of the substrate and the pressure transmitting media, Physical Review B 88 (4) (2013) 045418. doi:10.1103/ PhysRevB.88.045418.

[16] D. Yoon, H. Moon, Y. W. Son, J. S. Choi, B. H. Park, Y. H. e. a. Cha, Interference effect on Raman spectrum of graphene on $\mathrm{SiO} 2 / \mathrm{Si}$, Physical Review B - Condensed Matter and Materials Physics 80 (12) (2009) 1-21. doi:10.1103/PhysRevB.80.125422.

[17] Y. Y. Wang, Z. H. Ni, Z. X. Shen, H. M. Wang, $\mathrm{Y}$. H. Wu, Interference enhancement of raman signal of graphene, Applied Physics Letters 92 (4) (2008) 043121. doi : 10.1063/1.2838745.

[18] L. M. Malard, M. A. Pimenta, G. Dresselhaus, M. S. Dresselhaus, Raman spectroscopy in graphene, Physics Reports 473 (5-6) (2009) 51-87. doi:10.1016/j. physrep.2009.02.003.

[19] A. C. Ferrari, J. C. Meyer, V. Scardaci, C. Casiraghi, M. Lazzeri, F. e. a. Mauri, Raman spectrum of graphene and graphene layers, Phys. Rev. Lett. 97 (2006) 187401. doi:10.1103/PhysRevLett.97.187401.

[20] J. E. Proctor, E. Gregoryanz, K. S. Novoselov, M. Lotya, J. N. Coleman, M. P. Halsall, High-pressure 
Raman spectroscopy of graphene, Physical Review B 80 (7) (2009) 073408. doi:10.1103/PhysRevB.80. 073408.

[21] R. S. Alencar, K. D. A. Saboia, D. Machon, G. Montagnac, V. Meunier, O. P. e. a. Ferreira, Atomic-layered MoS2 on $\mathrm{SiO} 2$ under high pressure: Bimodal adhesion and biaxial strain effects, Physical Review Materials 1 (2) (2017) 024002. doi:10.1103/PhysRevMaterials. 1.024002 .

[22] S. Deng, Wrinkled, rippled and crumpled graphene: an overview of formation mechanism, electronic properties, and applications, Materials Today 19 (4) (2016) 16. doi:10.1016/j.mattod.2015.10.002.

[23] C. Androulidakis, E. N. Koukaras, O. Frank, G. Tsoukleri, D. Sfyris, J. e. a. Parthenios, Failure Processes in Embedded Monolayer Graphene under Axial Compression, Scientific Reports 4 (1) (2015) 5271. doi: 10.1038/srep05271.

[24] D. Yoon, Y.-W. Son, H. Cheong, Negative Thermal Expansion Coefficient of Graphene Measured by Raman Spectroscopy, Nano Letters 11 (8) (2011) 3227-3231. doi:10.1021/nl201488g.

[25] T. Jiang, Z. Wang, X. Ruan, Y. Zhu, Equi-biaxial compressive strain in graphene: Grüneisen parameter and buckling ridges, 2D Materials 6 (1) (2018) 015026. doi:10.1088/2053-1583/aaf20a.

[26] E. N. Koukaras, C. Androulidakis, G. Anagnostopoulos, K. Papagelis, C. Galiotis, Compression behavior of simply-supported and fully embedded monolayer graphene: Theory and experiment, Extreme Mechanics Letters 8 (2016) 191-200. doi:10.1016/j.eml.2016. 03.016 .

[27] Y. Magnin, F. Rondepierre, W. Cui, D. Dunstan, A. San-Miguel, Collapse phase diagram of carbon nanotubes with arbitrary number of walls. collapse modes and macroscopic analog, Carbon 178 (2021) 552562. doi:https://doi.org/10.1016/j. carbon. 2021. 03.031.

[28] P. Blake, E. W. Hill, A. H. Castro Neto, K. S. Novoselov, D. Jiang, R. e. a. Yang, Making graphene visible, Applied Physics Letters 91 (6) (2007) 063124. doi:10.1063/1.2768624.

[29] I. R. Amaral, R. S. Alencar, W. Paraguassu, D. A.
Costa, L. A. Costa, L. A. M. et al, Structural and vibrational properties of carbonophosphates: Na3mco3po4 $(\mathrm{m}=\mathrm{mn}$, fe, co and ni), Spectrochimica Acta Part A: Molecular and Biomolecular Spectroscopy 223 (2019) 117298. doi:doi.org/10.1016/j.saa.2019.117298.

[30] K. S. Novoselov, A. K. Geim, S. V. Morozov, D. Jiang, Y. Zhang, S. V. e. a. Dubonos, Electric field effect in atomically thin carbon films, Science 306 (5696) (2004) 666-669. doi:10.1126/science.1102896.

[31] R. Letoullec, J. P. Pinceaux, P. Loubeyre, The membrane diamond anvil cell: A new device for generating continuous pressure and temperature variations, High Pressure Research 1 (1) (1988) 77-90. doi:10.1080/ 08957958808202482.

[32] A. Castellanos-Gomez, M. Buscema, R. Molenaar, V. Singh, L. Janssen, H. S. J. e. a. van der Zant, Deterministic transfer of two-dimensional materials by all-dry viscoelastic stamping, 2D Materials 1 (1) (2014) 011002. doi:10.1088/2053-1583/1/1/011002.

[33] A. D. Chijioke, W. J. Nellis, A. Soldatov, I. F. Silvera, The ruby pressure standard to $150 \mathrm{GPa}$, Journal of Applied Physics 98 (11) (2005) 114905. doi: 10.1063/1.2135877.

[34] C. Coulson, Valence, The Oxford University Press, 1965.

[35] R. J. Angel, M. Bujak, J. Zhao, G. D. Gatta, S. D. Jacobsen, Effective hydrostatic limits of pressure media for high-pressure crystallographic studies, Journal of Applied Crystallography 40 (1) (2007) 26-32. doi: 10.1107/S0021889806045523.

[36] S. Klotz, J. C. Chervin, P. Munsch, G. Le Marchand, Hydrostatic limits of 11 pressure transmitting media, Journal of Physics D: Applied Physics 42 (7) (2009) 075413. doi:10.1088/0022-3727/42/7/075413.

[37] G. J. Piermarini, S. Block, J. D. Barnett, Hydrostatic limits in liquids and solids to $100 \mathrm{kbar}$, Journal of Applied Physics 44 (12) (1973) 5377-5382. doi:10.1063/ 1.1662159 .

[38] O. B. Tsiok, V. V. Brazhkin, A. G. Lyapin, L. G. Khvostantsev, Logarithmic kinetics of the amorphousamorphous transformations in $\mathrm{sio}_{2}$ and $\mathrm{geO}_{2}$ glasses under high pressure, Phys. Rev. Lett. 80 (1998) 999-1002. doi:10.1103/PhysRevLett.80.999. 
[39] F. Decremps, L. Belliard, M. Gauthier, B. Perrin, Equation of state, stability, anisotropy and nonlinear elasticity of diamond-cubic (zb) silicon by phonon imaging at high pressure, Physical Review B 82 (2010) 104119. doi:10.1103/PhysRevB.82.104119.

[40] P. W. Bridgman, The phase diagram of water to 45,000 $\mathrm{kg} / \mathrm{cm} 2$, The Journal of Chemical Physics 5 (12) (1937) 964-966. doi:10.1063/1.1749971.

[41] W. L. Vos, J. A. Schouten, Improved phase diagram of nitrogen up to $85 \mathrm{kbar}$, The Journal of Chemical Physics 91 (10) (1989) 6302-6305. doi:10.1063/1. 457397.

[42] J. W. Otto, J. K. Vassiliou, G. Frommeyer, Nonhydrostatic compression of elastically anisotropic polycrystals. i. hydrostatic limits of 4:1 methanol-ethanol and paraffin oil, Phys. Rev. B 57 (1998) 3253-3263. doi:10.1103/PhysRevB.57.3253.

[43] P. Gold, A. Schmidt, H. Dicke, J. Loos, C. Assmann, Viscosity-pressure-temperature behaviour of mineral and synthetic oils, Journal of Synthetic Lubrication 18 (2001) 51 - 79. doi:10.1002/jsl.3000180105.

[44] J. F. Mammone, S. K. Sharma, M. Nicol, Raman spectra of methanol and ethanol at pressures up to $100 \mathrm{kbar}$, Journal of Physical Chemistry 84 (23) (1980) 31303134. doi:10.1021/j100460a032.

[45] M. V. Kondrin, A. A. Pronin, Y. B. Lebed, V. V. Brazhkin, Phase transformations in methanol at high pressure measured by dielectric spectroscopy technique, Journal of Chemical Physics 139 (8) (2013) 084510. doi:10.1063/1.4819330.

[46] J. M. Zaug, L. J. Slutsky, J. M. Brown, Equilibrium properties and structural relaxation in methanol to 30.4 GPa, Journal of physical chemistry 98 (23) (1994) 60086016. doi:10.1021/j100074a030.

[47] M. J. Brugmans, W. L. Vos, Competition between vitrification and crystallization of methanol at high pressure, The Journal of Chemical Physics 103 (7) (1995) 2661-2669. doi:10.1063/1.470526.

[48] D. R. Allan, S. J. Clark, M. J. P. Brugmans, G. J. Ackland, W. L. Vos, Structure of crystalline methanol at high pressure, Phys. Rev. B 58 (1998) R11809-R11812. doi:10.1103/PhysRevB.58.R11809.

[49] R. Zangi, D. Roccatano, Strings-to-Rings Transition and Antiparallel Dipole Alignment in Two-Dimensional Methanols, Nano Letters 16 (5) (2016) 3142-3147. doi: 10.1021/acs. nanolett. 6 b00460.

[50] K. Morishige, K. Kawamura, A. Kose, X-ray diffraction study of the structure of a monolayer methanol film adsorbed on graphite, The Journal of Chemical Physics 93 (7) (1990) 5267-5270. doi:10.1063/1.459645.

[51] K. Morishige, Structure and melting of a monolayer ethanol film on graphite, The Journal of Chemical Physics 97 (3) (1992) 2084-2089. doi:10.1063/1. 463146.

[52] R. Zangi, Self-Assembly of Alcohols Adsorbed on Graphene, Journal of Physical Chemistry C 123 (27) (2019) 16902-16910. doi:10.1021/acs.jpcc.9b04839.

[53] E. del Corro, M. Peña-Álvarez, M. Mračko, R. Kolman, M. Kalbáč, L. a. a. Kavan, Graphene under direct compression: Stress effects and interlayer coupling, Physica Status Solidi (B) Basic Research 253 (12) (2016) 23362341. doi:10.1002/pssb. 201600202.

[54] M. Hanfland, H. Beister, K. Syassen, Graphite under pressure equation of state and first-order raman modes, Physical Review B 39 (1989) 17. doi:10.1103/ PhysRevB.39.12598.

[55] E. Han, J. Yu, E. Annevelink, J. Son, D. Kang, K. e. a. Watanabe, Ultrasoft slip-mediated bending in few-layer graphene, Nature Materials 19 (2020) 1-5. doi:10. 1038/s41563-019-0529-7.

[56] E. Schröder, Methanol adsorption on graphene, Journal of Nanomaterials 2013 (2013) 871706. doi:10.1155/ 2013/871706

[57] A. S. Bolina, A. J. Wolff, W. A. Brown, Reflection absorption infrared spectroscopy and temperature programmed desorption investigations of the interaction of methanol with a graphite surface, Journal of Chemical Physics 122 (4) (2005) 044713. doi:10.1063/1. 1839554.

[58] G. Wang, Z. Dai, J. Xiao, S. Feng, C. Weng, L. e. a. Liu, Bending of multilayer van der waals materials, Phys. Rev. Lett. 123 (2019) 116101. doi:10.1103/ PhysRevLett.123.116101.

[59] K. Zhang, M. Arroyo, Adhesion and friction control localized folding in supported graphene, Journal of Applied Physics 113 (19) (2013) 193501. doi:10.1063/1. 
4804265.

[60] B. Audoly, Stability of straight delamination blisters, Phys. Rev. Lett. 83 (1999) 4124-4127. doi:10.1103/ PhysRevLett.83.4124.

[61] D. Vella, J. Bico, A. Boudaoud, B. Roman, P. M. Reis, The macroscopic delamination of thin films from elastic substrates, Proceedings of the National Academy of Sciences 106 (27) (2009) 10901-10906. doi:10.1073/ pnas. 0902160106.

[62] L. Gong, R. J. Young, I. A. Kinloch, S. J. Haigh, J. H. Warner, J. A. e. a. Hinks, Reversible loss of bernal stacking during the deformation of few-layer graphene in nanocomposites, ACS Nano 7 (8) (2013) 7287-7294. doi:10.1021/nn402830f.

[63] S. P. Koenig, N. G. Boddeti, M. L. Dunn, J. S. Bunch, Ultrastrong adhesion of graphene membranes, Nature Nanotechnology 6 (9) (2011) 543. doi:10.1038/nnano. 2011.123.

[64] S. Kang, T. Yoon, B. S. Ma, M. S. Cho, T.-S. Kim, Liquid-assisted adhesion control of graphene-copper interface for damage-free mechanical transfer, Applied Surface Science (2021) 149229doi:10.1016/j.apsusc. 2021. 149229.

[65] A. C. Torres-Dias, T. F. Cerqueira, W. Cui, M. A. Marques, S. Botti, D. M. et al, From mesoscale to nanoscale mechanics in single-wall carbon nanotubes, Carbon 123 (2017) 145-150. doi:https://doi.org/10. 1016/j.carbon.2017.07.036.

[66] S. Liu, K. Duan, L. Li, X. Wang, Y. Hu, A multilayer coarse-grained molecular dynamics model for mechanical analysis of mesoscale graphene structures, Carbon 178 (2021) 528-539. doi:https://doi.org/10.1016/j . carbon.2021.03.025.

[67] P. Lambin, Elastic properties and stability of physisorbed graphene, Applied Sciences 4 (2) (2014) 282304. doi:10.3390/app4020282.

[68] B. Sajadi, S. van Hemert, B. Arash, P. Belardinelli, P. G. Steeneken, F. Alijani, Size- and temperaturedependent bending rigidity of graphene using modal analysis, Carbon 139 (2018) 334-341. doi:https:// doi.org/10.1016/j.carbon.2018.06.066.

[69] G. J. Fechine, I. Martin-Fernandez, G. Yiapanis, R. Bentini, E. S. Kulkarni, R. V. B. de Oliveira et al, Direct dry transfer of chemical vapor deposition graphene to polymeric substrates, Carbon 83 (2015) 224-231. doi:https://doi.org/10.1016/j.carbon. 2014.11 .038 .

[70] W. Zhai, N. Srikanth, L. B. Kong, K. Zhou, Carbon nanomaterials in tribology, Carbon 119 (2017) 150171. doi:https://doi.org/10.1016/j.carbon. 2017. 04.027 .

[71] F. Pape, G. Poll, Investigations on graphene platelets as dry lubricant and as grease additive for sliding contacts and rolling bearing application, Lubricants 8 (1) (2020) 1. doi:10.3390/lubricants8010003.

[72] T. Le Goff, T. B. T. To, O. Pierre-Louis, Shear dynamics of confined membranes, Soft Matter 17 (2021) 5467-5485. doi:10.1039/D1SM00322D.

[73] O. V. Sedelnikova, L. G. Bulusheva, A. V. Okotrub, Curvature-induced optical transitions in graphene, Fullerenes, Nanotubes and Carbon Nanostructures 20 (4-7) (2012) 558-562. doi:10.1080/1536383X. 2012. 656486.

[74] J. Sabalot-Cuzzubbo, G. Salvato-Vallverdu, D. Bégué, J. Cresson, Relating the molecular topology and local geometry: Haddon's pyramidalization angle and the gaussian curvature, The Journal of Chemical Physics 152 (24) (2020) 244310. doi:10.1063/5.0008368. 